\title{
RELAXATION OF SINGULAR FUNCTIONALS DEFINED ON SOBOLEV SPACES
}

\author{
Hafedh Ben BelgaCEM ${ }^{1}$
}

\begin{abstract}
In this paper, we consider a Borel measurable function on the space of $m \times n$ matrices $f: M^{m \times n} \rightarrow \overline{\mathbb{R}}$ taking the value $+\infty$, such that its rank-one-convex envelope $R f$ is finite and satisfies for some fixed $p>1$ :

$$
-c_{0} \leq R f(F) \leq c\left(1+\|F\|^{p}\right) \text { for all } F \in M^{m \times n},
$$
\end{abstract}

where $c, c_{0}>0$. Let $\Omega$ be a given regular bounded open domain of $\mathbb{R}^{n}$. We define on $W^{1, p}\left(\Omega ; \mathbb{R}^{m}\right)$ the functional

$$
I(u)=\int_{\Omega} f(\nabla u(x)) d x .
$$

Then, under some technical restrictions on $f$, we show that the relaxed functional $\bar{I}$ for the weak topology of $W^{1, p}\left(\Omega ; \mathbb{R}^{m}\right)$ has the integral representation:

$$
\bar{I}(u)=\int_{\Omega} Q[R f](\nabla u(x)) d x,
$$

where for a given function $g, Q g$ denotes its quasiconvex envelope.

Résumé. On considère une fonction Borel mesurable $f: M^{m \times n} \rightarrow \overline{\mathbb{R}}$ qui prend la valeur $+\infty$, dont l'enveloppe rang-1-convexe $R f$ est finie et satisfait pour un certain $p>1,-c_{0} \leq R f(F)$ $\leq c\left(1+\|F\|^{p}\right), \forall F \in M^{m \times n}$, avec $c, c_{0}>0$ fixés. Étant donné un ouvert borné $\Omega$ de $\mathbb{R}^{n}$, on introduit la fonctionnelle $I(u):=\int_{\Omega} f(\nabla u(x)) d x$, pour $u \in W^{1, p}\left(\Omega ; \mathbb{R}^{m}\right)$. On montre alors sous quelques hypothèses supplémentaires concernant $f$, que la relaxée $\bar{I}$ de $I$ pour la topolgie faible de $W^{1, p}\left(\Omega ; \mathbb{R}^{m}\right)$ admet la représentation suivante : $\bar{I}(u)=\int_{\Omega} Q R f(\nabla u(x)) d x$, où pour une fonction donnée $g, Q g$ désigne son enveloppe quasi-convexe.

AMS Subject Classification. 49-xx.

Received December 1, 1998. Revised November 17, 1999.

\section{INTRODUCTION}

Let $n, m \geq 1$ be two integers, $M^{m \times n}$ be the space of $m \times n$ real matrices endowed with the topology of $\mathbb{R}^{n m}$, and $\Omega \subset \mathbb{R}^{n}$ be an open bounded subset. Let $f: M^{m \times n} \rightarrow \overline{\mathbb{R}}$ be a Borel measurable function taking the value

Keywords and phrases: Rank-one convexity, quasiconvexity, weak lower semicontinuity.

1 Département de Mathématiques, Institut Préparatoire aux Études d'Ingénieurs de Sfax, Route Menzel Chaker - Km 0,5, BP. 805, 3000 Sfax, Tunisia; Fax: (00-216) 4. 246. 347.

Present address: Max-Planck Institute for Mathematics in the Sciences, Inselstr. 22-26, 04103 Leipzig, Germany; e-mail: Hafedh.Belgacem@mis.mpg.de 
$+\infty$, such that its rank-one-convex envelope $R f$ is everywhere finite. We introduce the functional

$$
I(u)=\int_{\Omega} f(\nabla u(x)) d x \text { for } u \in W^{1, p}\left(\Omega ; \mathbb{R}^{m}\right) .
$$

This functional is singular in the following sense: since $f$ takes the value $+\infty$ and its rank-one-convex envelope $R f$ is everywhere finite, it follows that $f$ cannot be rank-one-convex. Thus, using a result due to Tartar [30], we conclude that $I$ is not sequentially weakly lower semi-continuous on $W^{1, p}\left(\Omega ; \mathbb{R}^{m}\right)$.

It is usual in the calculus of variations to introduce the relaxed functional associated with $I$. In the case when $f$ is everywhere finite and satisfies the bound from below and growth assumption

$$
-c_{0} \leq f(F) \leq c\left(1+\|F\|^{p}\right) \text { for all } F \in M^{m \times n},
$$

Acerbi and Fusco [3] showed that the relaxed functional $\bar{I}$ of $I$ admits the integral representation

$$
\bar{I}(u)=\int_{\Omega} Q f(\nabla u(x)) d x, \forall u \in W^{1, p}\left(\Omega ; \mathbb{R}^{m}\right) .
$$

Let us emphasize that this formula is only applicable in the finite case, see Ball and Murat [5] for a counterexample with functions that take the value $+\infty$. More precisely, in the general case i.e., when $f$ takes the value $+\infty$, it is known that $Q f$ may give rise to a functional that is not weakly lower semi-continuous, see the above counter-example.

Our main result is that if $f$ takes the value $+\infty$ but $R f$ is everywhere finite and satisfies (1.1), then the relaxed functional admits the integral representation:

$$
\bar{I}(u)=\int_{\Omega} Q[R f](\nabla u(x)) d x
$$

under a few additional technical restrictions. In the proof, the main difficulty is to show that

$$
\bar{I}(u) \leq \int_{\Omega} R f(\nabla u(x)) d x, \text { for all piecewise affine functions. }
$$

We essentially use the results of Ball and Murat [5], Fonseca [16] and a characterization of the rank-one-convex envelope $R f$ due to Kohn and Strang $[19,20]$ to prove formula (1.2).

In Section 4, we will be concerned with the one-dimensional case. We thus recover the result of Acerbi et al. [1], and we give a generalization dealing with functions $f: \mathbb{R}^{m} \longrightarrow \overline{\mathbb{R}}$, such that the convex envelope $f^{* *}$ satisfies for some $1<q \leq p$, the following assumption:

$$
c^{\prime}|z|^{q}-c_{0} \leq f^{* *}(z) \leq c\left(1+|z|^{p}\right), \forall z \in \mathbb{R}^{m}
$$

where $c, c^{\prime}, c_{0}>0$ are fixed.

In Section 5, we give some applications to nonlinear elasticity that have to do with the stored-energy density for membranes $(n=2, m=3)$ and strings $(n=1, m=3)$.

Let us recall that the main impetus for all of those models was provided by the paper of Acerbi et al. [1]. These authors deal with strings, i.e., one-dimensional models, and use the tools of $\Gamma$-convergence theory to derive their result from genuine three-dimensional elasticity.

For two-dimensional models, Le Dret and Raoult $[21,22]$ used $\Gamma$-convergence techniques to obtain a nonlinear membrane model from a three dimensional hyperelastic body, whose stored energy function $W: M^{3 \times 3} \longrightarrow \mathbb{R}$ is everywhere finite. They showed that the stored energy function of the membrane is of the form $Q W_{0}$, where $W_{0}: M^{3 \times 2} \longrightarrow \mathbb{R}$ is deduced from the function $W$. 
Here, we will be primarily concerned with the two-dimensional case, and we give a generalization to the case: $W: M^{3 \times 3} \longrightarrow \overline{\mathbb{R}}$ satisfying the natural assumption: $W(F)=+\infty$ if and only if $\operatorname{det} F \leq 0$.

We show that using our relaxation result, the stored energy function of the membrane that we obtain is equal to $Q\left[R W_{0}\right]$. Here the auxiliary function $W_{0}: M^{3 \times 2} \longrightarrow \overline{\mathbb{R}}$ is such that $W_{0}(F)=+\infty$ if and only if $\operatorname{rank}(F)<2$.

We close this paper by showing how the study developed in Section 4 allow us to consider the case of strings for a large class of hyperelastic materials, namely those of Ogden [28].

\section{Preliminaries}

Throughout this section $g: M^{m \times n} \longrightarrow \overline{\mathbb{R}}$ is a Borel measurable, bounded below function.

Definition 2.1. The function $g$ is said to be quasiconvex if for all $F \in M^{m \times n}$

$$
g(F) \leq \frac{1}{\operatorname{meas}(D)} \int_{D} g(F+\nabla \phi(x)) d x
$$

for every bounded open set $D \subset \mathbb{R}^{n}$ with meas $(\partial D)=0$, and for all $\phi \in W_{0}^{1, \infty}\left(D ; \mathbb{R}^{m}\right)$.

For $a \in \mathbb{R}^{m}$ and $b \in \mathbb{R}^{n}$, we denote by $a \otimes b$ the rank-one-matrix $\left(a_{i} b_{j}\right)_{1 \leq i \leq m, 1 \leq j \leq n}$.

Definition 2.2. The function $g$ is said to be rank-one-convex if for all $F \in M^{m \times n}$

$$
g(F) \leq(1-\lambda) g(F-\lambda a \otimes b)+\lambda g(F+(1-\lambda) a \otimes b)
$$

for all $\lambda \in[0,1], a \in \mathbb{R}^{n}$ and $b \in \mathbb{R}^{m}$.

It is well known that rank-one-convexity follows from quasiconvexity for finite functions, see Dacorogna [1113] and Morrey [25, 26]. Ball and Murat [5] remarked that this is still true for continuous bounded below functions attaining the value $+\infty$. In addition, let us define for a function $g$, the effective domain to be

$$
\mathcal{D}_{e}(g):=\left\{F \in M^{m \times n} ; g(F) \text { is finite }\right\} .
$$

In [16], Fonseca showed,

Theorem 2.3. Let $g: M^{m \times n} \longrightarrow \overline{\mathbb{R}}$ be Borel measurable, bounded below and quasiconvex. Then $g$ is rank-oneconvex at every matrix $F$ belonging to the interior of its effective domain.

Remark. As a consequence of this theorem, it follows that a quasiconvex function is continuous on the interior of its effective domain. Thus, using Corollary 3.2. in Dacorogna and Marcellini [14], and Proposition 2 in Ball and James [6], one can show that the interior of the effective domain of a quasiconvex function is lamination convex. Let us recall that a set $K$ is lamination convex if for every $A, B \in K$ such that $\operatorname{rank} A-B \leq 1$, then $(1-\lambda) A+\lambda B \in K, \forall \lambda \in[0,1]$.

We now define the quasiconvex (resp. rank-one-convex) envelope, and we denote by $Q g$ (resp. $R g$ ), the largest quasiconvex (resp. rank-one-convex) function less than or equal to $g$. The quasiconvex envelope $Q g$ of a continuous function $g: M^{m \times n} \longrightarrow \mathbb{R}$ admits a representation obtained by Dacorogna [12, 13]. More precisely:

Theorem 2.4. Let $g: M^{m \times n} \longrightarrow \mathbb{R}$ be continuous and bounded below. If $D \subset \mathbb{R}^{n}$ is an open bounded set with meas $(\partial D)=0$ then

$$
Q g(F)=\inf \left\{\frac{1}{m e a s(D)} \int_{D} g(F+\nabla \phi(x)) d x ; \phi \in W_{0}^{1, \infty}\left(D ; \mathbb{R}^{m}\right)\right\}
$$

for all $F \in M^{m \times n}$. 
We do not know whether an analog of Theorem 2.4 holds for Borel measurable functions attaining the value $+\infty$. Let us define

$$
Z_{D} g(F):=\inf \left\{\frac{1}{\operatorname{meas}(D)} \int_{D} g(F+\nabla \phi(x)) d x ; \phi \in W_{0}^{1, \infty}\left(D ; \mathbb{R}^{m}\right)\right\}
$$

where $F \in M^{m \times n}$ and $D \subset \mathbb{R}^{m}$ is an open bounded set with meas $(\partial D)=0$. If $D=[0,1]^{n}$ then $Z_{D} g$ is denoted by $Z g$. Since

$$
Q g \leq Z_{D} g \leq g,
$$

(2.3) is satisfied if and only if $Z_{D} g$ is quasiconvex.

Following Ball and Murat [5], it is easy to show that:

Lemma 2.5. We have:

$$
Z_{D} g(F)=Z g(F) \text { for all } F \in M^{m \times n} .
$$

In the case when $g: M^{m \times n} \longrightarrow \overline{\mathbb{R}}$ is Borel measurable and bounded below, we do not know if $Z g$ is quasiconvex. Moreover, following Fonseca [16], we have

Lemma 2.6. Let $g$ be as above, then i) Let $D \subset \mathbb{R}^{n}$ be an open bounded set with meas $(\partial D)=0$. If $\xi \in$ $W_{0}^{1, \infty}\left(D ; \mathbb{R}^{m}\right)$ is a piecewise affine function then

$$
Z g(F) \leq \frac{1}{\operatorname{meas}(D)} \int_{D} Z g(F+\nabla \xi(x)) d x
$$

for all $F \in M^{m \times n}$.

ii) The function $Z g$ is rank-one-convex at every matrix $F \in \operatorname{int} \mathcal{D}_{e}(g)$.

Remark. Since the proofs of Lemmas 2.5 and 2.6 require the Vitali covering theorem, we are no longer sure that they are valid if in formula $(2.4)$, we replace $W_{0}^{1, \infty}\left(D ; \mathbb{R}^{m}\right)$ by the subclass of piecewise affine functions. A new class of functions will be needed that is larger than the set of piecewise affine functions.

Definition 2.7. Let $D \subset \mathbb{R}^{n}$ be an open bounded subset, a function $\phi \in W^{1, \infty}\left(D ; \mathbb{R}^{m}\right)$ is said to be Vitali piecewise affine on $D$ if and only if the following conditions are satisfied:

(i) $\operatorname{Card}\{\nabla u(y) ; y \in D\}$ is finite,

(ii) there exists a countable family of open disjoint subsets $\left(O_{i}\right)_{i \in I}$ with meas $\left(\partial O_{i}\right)=0$ for all $i \in I$ and meas $\left(D \backslash \cup_{i \in I} O_{i}\right)=0$, such that the restriction of $\phi$ to $O_{i}$ is affine.

As an example of such a function, let $D$ be an open subset of $\mathbb{R}^{n}$, and consider a piecewise affine function $u$ on the unit ball $B(0,1)$ with $u=0$ on $\partial B(0,1)$. By the Vitali covering theorem, there exists a finite or countable disjoint sequence $a_{i}+\varepsilon_{i} \bar{B}(0,1)$ of subsets of $D$, where $a_{i} \in \mathbb{R}^{n}$ and $0<\varepsilon_{i}<1$, such that

$$
\operatorname{meas}\left(D \backslash \bigcup_{i}\left(a_{i}+\varepsilon_{i} \bar{B}(0,1)\right)=0\right.
$$

Let us define on $D$, the function

$$
v(x):=\left\{\begin{array}{cc}
\varepsilon_{i} u\left(\frac{x-a_{i}}{\varepsilon_{i}}\right), & \text { if } x \in a_{i}+\varepsilon_{i} \bar{B}(0,1), \\
0, & \text { otherwise }
\end{array}\right.
$$

Clearly, the function $v$ thus constructed is Vitali piecewise affine on $D$.

We introduce for each bounded domain $D$, the subspace

$$
\mathcal{V}_{0}\left(D ; \mathbb{R}^{m}\right):=\{\phi \text { is Vitali piecewise affine on } D \text { and } \phi=0 \text { on } \partial D\}
$$


Now, let $g$ be a given function, we define

$$
Z_{D}^{V} g(F):=\inf \left\{\frac{1}{\operatorname{meas}(D)} \int_{D} g(F+\nabla \phi(x)) d x ; \phi \in \mathcal{V}_{0}\left(D ; \mathbb{R}^{m}\right)\right\}
$$

for each open bounded subset $D$ with meas $(\partial D)=0$. As in the above lemmas, we claim that

Proposition 2.8. The function $Z_{D}^{V}$ g satisfies the following:

i) Let $D \subset \mathbb{R}^{n}$ be an open bounded set with meas $(\partial D)=0$, then $Z_{D}^{V} g(F)=Z^{V} g(F)$ for all $F \in M^{m \times n}$.

ii) Let $D \subset \mathbb{R}^{n}$ be an open bounded set with meas $(\partial D)=0$. If $\xi \in W_{0}^{1, \infty}\left(D ; \mathbb{R}^{m}\right)$ is a Vitali piecewise affine function, then

$$
Z^{V} g(F) \leq \frac{1}{\operatorname{meas}(D)} \int_{D} Z^{V} g(F+\nabla \xi(x)) d x
$$

for all $F \in M^{m \times n}$.

iii) $Z^{V} g$ is rank-one-convex on the interior of its effective domain.

Proof. We only sketch it, since part i) follows exactly the lines of Ball and Murat [5] Proposition 2.3. Part ii) is based on the Vitali covering theorem. Finally, for part iii), an examination of the details of the proof of the Theorem 2.4 in Fonseca [16] shows that the conclusion wil remain valid if quasiconvexity is replaced by condition (2.8).

Remark. We see at once that $Z^{V} g$ is larger than Dacorogna's function $Z g$. However, since $Z^{V} g$ is less then $g$, its effective domain may be greater than the one of $g$. Therefore, the set where $Z^{V} g$ is rank-one-convex is larger than the one of $Z g$.

Now, we recall an algorithm due to Kohn and Strang [19, 20] for computing the rank-one-convex envelope of a function.

Proposition 2.9. Let $g$ be a Borel measurable function and bounded below. Define the sequence $\left(R_{i}(g)\right)_{i \in \mathbb{N}}$ by $R_{0} g=g$ and

$$
R_{i+1} g(F)=\inf \left\{(1-\lambda) R_{i} g(F-\lambda a \otimes b)+\lambda R_{i} g(F+(1-\lambda) a \otimes b) ; \text { for all } \lambda \in[0,1], a \in \mathbb{R}^{m} \text { and } b \in \mathbb{R}^{n}\right\},
$$

for $i \geq 1$.

Then for all $F \in M^{m \times n}$, the sequence $\left(R_{i} g(F)\right)_{i \in \mathbb{N}}$ decreases to $R g(F)$.

Finally, we give a characterization of $Q[R f]$ for a function $f$ whose rank-one-convex envelope is everywhere finite.

Let $g: M^{m \times n} \longrightarrow \overline{\mathbb{R}}$ be Borel measurable and bounded below, $\Omega \subset \mathbb{R}^{n}$ be an open bounded domain with regular bound.

We consider for some fixed $p>1$, the functional

$$
J(u):=\int_{\Omega} g(\nabla u(x)) d x
$$

for all $u \in W^{1, p}\left(\Omega ; \mathbb{R}^{m}\right)$. Ball and Murat [5] showed,

Theorem 2.10. Let us suppose that $J$ is sequentially weakly lower semi-continuous on $W^{1, p}\left(\Omega ; \mathbb{R}^{m}\right)$. Then $g$ is lower semi-continuous, and

$$
g\left(\frac{1}{\operatorname{meas}(Q)} \int_{Q} \nabla v(x) d x\right) \leq \frac{1}{\operatorname{meas}(Q)} \int_{Q} g(\nabla v(x)) d x
$$

for every $n$-cube $Q$ and for all $v \in W_{\mathrm{loc}}^{1, p}\left(\mathbb{R}^{n} ; \mathbb{R}^{m}\right)$ such that $\nabla v$ is $Q$-periodic. 
Note that formula (2.10) implies that $g$ is at the same time quasiconvex and rank-one-convex. We thus claim,

Proposition 2.11. Let $f: M^{m \times n} \longrightarrow \overline{\mathbb{R}}$ be a Borel measurable, bounded below function taking the value $+\infty$, such that its rank-one-convex envelope $R f$ is everywhere finite and satisfies the following growth assumption:

$$
R f(F) \leq c\left(1+\|F\|^{p}\right) \text { for all } F \in M^{m \times n}
$$

where $c>0$ is fixed. Then,

$$
Q[R f](F)=\sup \{h(F) ; h \leq f \text { satisfying }(2.10)\}
$$

for all $F \in M^{m \times n}$.

\section{RelaxAtion}

Let $f: M^{m \times n} \rightarrow \overline{\mathbb{R}}$ be Borel measurable, bounded below with the following hypotheses:

$\left(\mathrm{H}_{1}\right)$ the open set $O_{f}:=\operatorname{int}\left\{F \in M^{m \times n} ; Z^{V}\left[R_{i} f\right](F) \leq R_{i+1} f(F)\right.$, for all $i$ integer $\}$ is dense in $M^{m \times n}$.

$\left(\mathrm{H}_{2}\right)$ For each $F \in M^{m \times n}$ and $i \geq 1$ fixed,

$$
\limsup _{\varepsilon \rightarrow 0^{+}} R_{i} f\left(F_{\varepsilon}\right) \leq R_{i} f(F),
$$

whenever $\left(F_{\varepsilon}\right)_{\varepsilon>0}$ belongs to $O_{f}, F_{\varepsilon} \longrightarrow F$ when $\varepsilon \rightarrow 0$.

$\left(\mathrm{H}_{3}\right)$ The rank-one-convex envelope $R f$ is everywhere finite and satisfies for some fixed $p>1$ the following growth assumption:

$$
R f(F) \leq c\left(1+\|F\|^{p}\right), \text { for all } F \in M^{m \times n},
$$

where $c>0$.

Let us consider the functional on $W^{1, p}\left(\Omega ; \mathbb{R}^{m}\right)$ with values in $\overline{\mathbb{R}}$

$$
I(u):=\int_{\Omega} f(\nabla u(x)) d x .
$$

We denote $\bar{I}$ its relaxed functional for the weak topology of $W^{1, p}\left(\Omega ; \mathbb{R}^{m}\right)$.

Let us now state our main result,

Theorem 3.1. Let $f$ satisfy $\left(H_{1}\right),\left(H_{2}\right)$ and $\left(H_{3}\right)$. Then the relaxed functional admits the integral representation

$$
\bar{I}(u)=\int_{\Omega} Q[R f](\nabla u(x)) d x .
$$

Remarks. (i) Here the quasiconvex envelope that is computed is not that of $f$ but that of the rank-one-convex envelope of $f$. Indeed, the quasiconvex envelope may give rise to a non lower semi-continuous functional. Moreover, when $f$ is everywhere finite, we can easily see that $Q[R f]=Q f$, and we thus recover Acerbi and Fusco's relaxation theorem [2].

(ii) A prototype example, which derives from nonlinear elasticity, is the case of strings $n=1, m=3$ and membranes $n=2, m=3$, where $f$ takes the value $+\infty$ only on the set of matrices $F \in M^{m \times n}$ such that $\operatorname{rank}(F)<n$.

(iii) The upper semicontinuity hypothesis $\left(\mathrm{H}_{2}\right)$ of the sequence of functions $\left(R_{i} f\right)_{i \in \mathbb{N}^{*}}$ can be satisfied, even if $f$ fails to be upper semi-continuous on $M^{m \times n}$. Indeed, only the upper semicontinuity of the restriction of $f$ to its effective domain $\mathcal{D}_{e}(f)$, together with some geometric considerations on the connected compenents of this set, can be sufficient to obtain upper semicontinuity of the sequence of the Kohn and Strang algorithm. 
(iv) In the restrictive technical condition $\left(\mathrm{H}_{1}\right)$ inequality is not strict, so the finiteness of $Z^{V}\left[R_{i} f\right]$ is not required. It guarantees the strong density in $W^{1, p}\left(\Omega ; \mathbb{R}^{m}\right)$, of the class $\{u$ is Vitali piecewise affine; $\nabla u(x) \in$ $O_{f}$ a.e. in $\Omega$ \}. More precisely, we have

Lemma 3.2. Let $(A)_{j \in J}$ be a finite family of matrices on $M^{m \times n}$, then for all $\eta>0$ there exists a matrix $F \in M^{m \times n}$ with $\|F\|<\eta$, such that

$$
A_{j}+F \in O_{f}, \text { for all } j \in J \text {. }
$$

Proof. Let $\left(A_{j}\right)_{j \in J}$ be a given family. We define for each $j \in J$, the open subset

$$
O_{j}:=-A_{j}+O_{f}
$$

Since $O_{f}$ is dense on $M^{m \times n}$, we infer that $O_{j}$ is dense for each $j \in J$. Let us now consider the intersection $\cap_{j \in J} O_{j}$. Thus, using a standard topological argument, we conclude that it is also dense on $M^{m \times n}$. And, in particular 0 is one of its limit points. This yields that for all $\eta>0$, their exists $F \in \cap_{j \in J} O_{j}$, such that $\|F\|<\eta$. From the definition of $\left(O_{j}\right)_{j \in J}$, we obtain that $A_{j}+F \in O_{f}$ for all $j \in J$.

Let us now give the proof of Theorem 3.1.

Proof. First of all, let us consider the functional

$$
J(u)=\int_{\Omega} Q[R f](\nabla u(x)) d x
$$

defined for all $u \in W^{1, p}\left(\Omega ; \mathbb{R}^{m}\right)$. It is easy to see that this functional is sequentially weakly lower semi-continuous on $W^{1, p}\left(\Omega ; \mathbb{R}^{m}\right)$ and below $I$. Therefore,

$$
\int_{\Omega} Q[R f](\nabla u(x)) d x \leq \bar{I}(u), \text { for all } u \in W^{1, p}\left(\Omega ; \mathbb{R}^{m}\right)
$$

Due to this inequality, the proof of (3.3) will be complete once we have shown the reverse inequality

$$
\bar{I}(u) \leq \int_{\Omega} Q[R f](\nabla u(x)) d x
$$

The proof follows in five steps.

Step 1. We claim that

Claim 3.3. For every Vitali piecewise affine function $u$ such that $\nabla u(x) \in O_{f}$ a.e. in $\Omega$, we have

$$
\bar{I}(u) \leq \int_{\Omega} R_{i} f(\nabla u(x)) d x
$$

for all integers $i$.

Proof of the claim. The proof is by induction on $i$.

First, let us recall that for $i=0, R_{0} f=f$ and there is nothing to show.

Now, let us assume that for some $i \geq 0$, formula (3.6) holds for each Vitali piecewise affine function $u$, such that $\nabla u(x) \in O_{f}$ a.e. $x \in \Omega$. Thus, we have to prove that it is still valid for $i+1$.

Let $u$ be as above, we denote by $\left(A_{j}\right)_{j \in J}$ the values of the gradient of $u$, and $\left(O_{k}\right)_{k \in K}$ the partition of $\Omega$ corresponding to $u$. We will also suppose that $R_{i+1} f\left(A_{j}\right)<\infty$, otherwise (3.6) is trivial. Let $\varepsilon>0$ be fixed, 
From the definition of $Z^{V}\left[R_{i} f\right]$ and Proposition 2.8, it follows that there exists a family of Vitali piecewise affine functions $\left(\phi_{j}\right)_{j \in J}$ belonging to $W_{0}^{1, \infty}\left(Q ; \mathbb{R}^{m}\right)$, where $Q$ denotes the unit open cube of $\mathbb{R}^{n}$, such that

$$
\frac{1}{\operatorname{meas}(Q)} \int_{Q} R_{i} f\left(A_{j}+\nabla \phi_{j}(x)\right) d x \leq Z^{V}\left[R_{i} f\right]\left(A_{j}\right)+\varepsilon, \text { for each } j \in J .
$$

For each $j \in J$, we extend $\phi_{j}$ by periodicity to $\mathbb{R}^{n}$ and define the sequence $\left(\phi_{j}(r)\right)_{r \geq 1}$ by

$$
\phi_{j}(r)(x):=r^{-1} \phi_{j}(r x), \forall x \in \mathbb{R}^{n} .
$$

Now, for each fixed $k \in K$, we recall that $u$ is affine on $O_{k}$, so there exists $j \in J$ such that $\nabla u(x)=A_{j}$ for almost all $x \in O_{k}$. By applying the Vitali covering theorem, we obtain that there exists a countable disjoint family $a_{k}^{s}+\alpha_{k}^{s} Q$ of subsets of $O_{k}$, where $a_{k}^{s} \in \mathbb{R}^{n}$ and $0<\alpha_{k}^{s}<1$, such that

$$
\text { meas }\left(O_{k} \backslash \bigcup_{s}\left(a_{k}^{s}+\alpha_{k}^{s} \bar{Q}\right)\right)=0 \text {. }
$$

Let $s$ be fixed, we consider on $a_{k}^{s}+\alpha_{k}^{s} Q$ the function

$$
\phi_{k, j}^{s}(r)(x):=\alpha_{k}^{s} \phi_{j}(r)\left(\frac{x-a_{k}^{s}}{\alpha_{k}^{s}}\right) .
$$

Let us now introduce on $\Omega$ the sequence $(\phi(r))_{r \in \mathbb{N}^{*}}$, as the following:

$$
\phi(r)(x):=\left\{\begin{array}{cc}
\phi_{k, j}^{s}(r)(x), & \text { if } x \in a_{k}^{s}+\alpha_{k}^{s} Q, \\
0, & \text { otherwise. }
\end{array}\right.
$$

Let us denote by $\phi=\phi(1)$, we remark that this constructed sequence of Vitali piecewise affine functions satisfies for all $r \in \mathbb{N}^{*}$ the following:

$$
\int_{\Omega} R_{i} f(\nabla u(x)+\nabla \phi(r)(x)) d x=\int_{\Omega} R_{i} f(\nabla u(x)+\nabla \phi(x)) d x \leq \int_{\Omega} Z^{V}\left[R_{i} f\right](\nabla u(x)) d x+\varepsilon \text { meas }(\Omega) .
$$

In addition, $\phi(r) \stackrel{*}{\rightarrow} 0$ in $W^{1, \infty}\left(\Omega ; \mathbb{R}^{m}\right)$ when $r \rightarrow+\infty$.

Now, we use Lemma 3.2 to conclude that, for each $\eta>0$ there exists a matrix $F_{\eta} \in B(0, \eta)$ such that:

$$
\nabla u(x)+\nabla \phi(x)+F_{\eta} \in O_{f} \text {, a.e } x \in \Omega .
$$

Let us denote by $L_{\eta}$ the linear map $L_{\eta}(x):=F_{\eta} x$, and consider for each fixed $r \in \mathbb{N}^{*}$, the Vitali piecewise affine function $\psi_{\eta}(r):=u+\phi(r)+L_{\eta}$. The induction argument yields:

$$
\bar{I}\left(\psi_{\eta}(r)\right) \leq \int_{\Omega} R_{i} f\left(\nabla \psi_{\eta}(r)(x)\right) d x
$$

Since the right hand side is constant in $r$, and equal to $\int_{\Omega} R_{i} f\left(\nabla u(x)+\nabla \phi(x)+F_{\eta}\right) d x$, we obtain that:

$$
\bar{I}\left(\psi_{\eta}(r)\right) \leq \int_{\Omega} R_{i} f\left(\nabla u(x)+\phi(x)+F_{\eta}\right) d x .
$$

By passing to the limit when $r \rightarrow+\infty$, we conclude that:

$$
\bar{I}\left(u+L_{\eta}\right) \leq \int_{\Omega} R_{i} f\left(\nabla u(x)+\nabla \phi(x)+F_{\eta}\right) d x,
$$


for all $\eta>0$. Now, to pass to the limit when $\eta \rightarrow 0$, we recall that for each fixed $\eta>0, \psi_{\eta}:=u+\phi+L_{\eta}$ is Vitali piecewise affine satisfying $\nabla \psi_{\eta} \in O_{f}$ a.e. $x \in \Omega$. This implies that the right hand side of last inequality is a convex combination whose members are the values of $R_{i} f$ on matrices belonging to $O_{f}$. Thus, using hypothesis $\left(\mathrm{H}_{2}\right)$ on $R_{i} f$, we conclude that:

$$
\begin{aligned}
\bar{I}(u) \leq \liminf _{\eta \rightarrow 0^{+}} \bar{I}\left(u+L_{\eta}\right) & \leq \limsup _{\eta \rightarrow 0^{+}} \bar{I}\left(u+L_{\eta}\right) \leq \limsup _{\eta \rightarrow 0^{+}} \int_{\Omega} R_{i} f\left(\nabla u(x)+\nabla \phi(x)+F_{\eta}\right) d x \\
& \leq \int_{\Omega} R_{i} f(\nabla u(x)+\nabla \phi(x)) d x .
\end{aligned}
$$

This combined with (3.8), implies

$$
\bar{I}(u) \leq \int_{\Omega} Z^{V}\left[R_{i} f\right](\nabla u(x)) d x+\varepsilon \operatorname{meas}(\Omega) .
$$

Now, let us recall that $\nabla u(x) \in O_{f}$ for almost all $x \in \Omega$, and in particular

$$
Z^{V}\left[R_{i} f\right](\nabla u(x)) \leq R_{i+1} f(\nabla u(x))
$$

for almost all $x \in \Omega$. We thus conclude that

$$
\bar{I}(u) \leq \int_{\Omega} R_{i+1} f(\nabla u(x)) d x+\varepsilon \operatorname{meas}(\Omega) .
$$

Now, due to the arbitrariness of $\varepsilon$, it follows that

$$
\bar{I}(u) \leq \int_{\Omega} R_{i+1} f(\nabla u(x)) d x
$$

for each Vitali piecewise affine function $u \in W^{1, \infty}\left(\Omega ; \mathbb{R}^{m}\right)$, such that $\nabla u(x)$ belongs to $O_{f}$ for a.e. $x \in \Omega$, which completes the proof of the claim.

Step 2. Let $u$ be as above, we assert that:

$$
\bar{I}(u) \leq \int_{\Omega} R f(\nabla u(x)) d x .
$$

Indeed, let $u$ be such a function, we recall that $\operatorname{Card}\{\nabla u(x), x \in \Omega\}$ is finite.

Now, taking account of this fact and the finiteness of the rank-one-convex envelope $R f$, we deduce with the use of the Kohn and Strang algorithm, that the decreasing sequence $\left(R_{i} f(\nabla u(.))\right)_{i \in \mathbb{N}}$ is everywhere finite from some $i_{0}$. Therefore, formula (3.9) follows by applying the Lebesgue monotone convergence theorem to (3.6).

Step 3. Let us now show that

for each Vitali piecewise affine function $u$.

$$
\bar{I}(u) \leq \int_{\Omega} R f(\nabla u(x)) d x
$$

Let $u$ be such a function.

First, from Lemma 3.2 we infer that there exists a sequence of Vitali piecewise affine functions $\left(u_{s}\right)_{s \in \mathbb{N}}$ with $\nabla u_{s}(x) \in O_{f}$ a.e. $x \in \Omega$ and $u_{s} \stackrel{s \rightarrow+\infty}{\longrightarrow} u$ in $W^{1, \infty}\left(\Omega ; \mathbb{R}^{m}\right)$. 
Second, by the previous step, we have

$$
\bar{I}\left(u_{s}\right) \leq \int_{\Omega} R f\left(\nabla u_{s}(x)\right) d x
$$

for all $s \in \mathbb{N}$.

To conclude, since $R f$ is everywhere finite and rank-one-convex, it follows with the use of a standard argument of convex analysis, that it is continuous, and thus

$$
\bar{I}(u) \leq \int_{\Omega} R f(\nabla u(x)) d x .
$$

Step 4. We claim that formula (3.10) holds for all $u \in W^{1, p}\left(\Omega ; \mathbb{R}^{m}\right)$.

Indeed, the growth assumption on the rank-one-convex envelope $R f$

$$
R f(F) \leq c\left(1+\|F\|^{p}\right), \text { for all } F \in M^{m \times n}
$$

implies that the right hand side of $(3.10)$ is continuous on $W^{1, p}\left(\Omega ; \mathbb{R}^{m}\right)$ endowed with its strong topology.

To conclude, we thus only have to use the density of the class of Vitali piecewise affine functions in $W^{1, p}\left(\Omega ; \mathbb{R}^{m}\right)$ together with the dominated convergence theorem.

Step 5. The proof of formula (3.5) is an immediate consequence of Acerbi and Fusco's relaxation theorem applied together with the dominated convergence theorem, to the functional

$$
u \mapsto \int_{\Omega} R f(\nabla u(x)) d x .
$$

Remarks. Let us make a few more comments about the hypotheses of the theorem.

(i) Since for the range $i=1$, the proof of formula (3.6) is the same as of the preceeding, without the use of hypothesis $\left(\mathrm{H}_{2}\right)$, so in hypothesis $\left(\mathrm{H}_{1}\right)$ one can take $O_{f}$ as the following:

$$
\operatorname{int}\left\{F \in M^{m \times n} ; Z f(F) \leq R_{1} f(F)\right\} \cap \operatorname{int}\left\{F \in M^{m \times n} ; Z^{V}\left[R_{i} f\right](F) \leq R_{i+1} f(F) \text { for each } i \geq 1\right\}
$$

where $Z f$ denotes Dacorogna's function.

(ii) Hypothesis $\left(\mathrm{H}_{2}\right)$ is not needed if $O_{f}$ is equal to $M^{m \times n}$.

\section{The One-Dimensional CASE}

Let us take $n=1$ and $p>1$, and consider a Borel measurable function $f: \mathbb{R}^{m} \rightarrow \overline{\mathbb{R}}$, such that its convex envelope is everywhere finite and satisfies for some $c, c_{0}>0$ the bound from below and growth assumption:

$$
-c_{0} \leq f^{* *}(z) \leq c\left(1+|z|^{p}\right) \text {, for all } z \in \mathbb{R}^{m}
$$

Here, since $n=1$, we do not need to impose the restrictive conditions $\left(\mathrm{H}_{1}\right)$ and $\left(\mathrm{H}_{2}\right)$, to apply our relaxation result, and we thus generalize Acerbi et al.'s result.

Now, we give a more general result. Let us consider a Borel measurable function $f: \mathbb{R}^{m} \longrightarrow \overline{\mathbb{R}}$, such that its convex envelope $f^{* *}$ satisfies for some fixed $1<q \leq p$, the following growth and coercivity hypotheses:

$$
c^{\prime}|z|^{q}-c_{0} \leq f^{* *}(z) \leq c\left(1+|z|^{p}\right)
$$


where $c, c^{\prime}, c_{0}>0$ are a given constants. As in Marcellini [24] and Fonseca [17], we introduce:

$$
\tilde{I}(u)=\inf \left\{\liminf _{s \rightarrow 0} \int_{0}^{1} f\left(u_{s}^{\prime}(x)\right) d x \mid u_{s} \in W^{1, p}(] 0,1\left[; \mathbb{R}^{m}\right), u_{s} \rightarrow u \text { in } W^{1, q}(] 0,1\left[; \mathbb{R}^{m}\right)\right\} .
$$

Let us state the main result of this section:

Theorem 4.1. Let $f$ be as above, then the relaxed functional admits the integral representation:

$$
\tilde{I}(u)=\int_{0}^{1} f^{* *}\left(u^{\prime}(x)\right) d x, \text { for all } u \in W^{1, q}(] 0,1\left[; \mathbb{R}^{m}\right) .
$$

Remarks. (i) Here the relaxation is given with respect to the exponent of coerciveness, which is coherent with the corresponding minimization problem.

(ii) The result obtained here is a special case of a more general one etablished by Fonseca and Malý in [18 ]. Before proving the theorem, we first consider the functional:

$$
J(u):=\int_{0}^{1} f^{* *}\left(u^{\prime}(x)\right) d x
$$

defined for every $u \in W^{1, q}(] 0,1\left[; \mathbb{R}^{m}\right)$.

Following Marcellini [24], we have:

Proposition 4.2. If (4.2) holds, then for each $u \in W^{1, q}(] 0,1\left[; \mathbb{R}^{m}\right)$, we have

$$
J(u) \leq \liminf _{s \rightarrow+\infty} J\left(u_{s}\right),
$$

whenever $\left(u_{s}\right)_{s \in \mathbb{N}} \subset W^{1, p}(] 0,1\left[; \mathbb{R}^{m}\right)$, such that $u_{s} \rightarrow u$ in $W^{1, q}(] 0,1\left[; \mathbb{R}^{m}\right)$ as $s \rightarrow+\infty$.

As an immediate consequence of the last proposition, we obtain that

$$
\int_{0}^{1} f^{* *}\left(u^{\prime}(x)\right) d x \leq \tilde{I}(u)
$$

for all $u \in W^{1, q}(] 0,1\left[; \mathbb{R}^{m}\right)$.

Thus, the proof of the theorem is complete once we show:

Proposition 4.3. Let $f$ be as above, then:

$$
\tilde{I}(u) \leq \int_{0}^{1} f^{* *}\left(u^{\prime}(x)\right) d x
$$

for each $u \in W^{1, q}(] 0,1\left[; \mathbb{R}^{m}\right)$.

The proof of formula (4.7) is based on the following lemma:

Lemma 4.4. Let $h: \mathbb{R}^{m} \longrightarrow \mathbb{R}$ be convex and bounded below. Then, for each $u \in W^{1,1}(] 0,1\left[; \mathbb{R}^{m}\right)$ satisfying $\int_{0}^{1} h\left(u^{\prime}(x)\right) d x$ is finite, there exists a sequence $\left(u_{s}\right)_{s \in \mathbb{N}^{*}} \subset C^{\infty}\left([0,1] ; \mathbb{R}^{m}\right)$, such that when $s \rightarrow+\infty$ :

$$
\left\|u_{s}-u\right\|_{W^{1,1}(] 0,1\left[; \mathbb{R}^{m}\right)} \longrightarrow 0, \int_{0}^{1} h\left(u_{s}^{\prime}(x)\right) d x \longrightarrow \int_{0}^{1} h\left(u^{\prime}(x)\right) d x .
$$


See Ekeland et Temam [15] for the proof. Note that the conclusion of this lemma also holds in $W^{1, q}(] 0,1\left[; \mathbb{R}^{m}\right)$ for each $q \in] 1,+\infty[$.

Proof of Proposition 4.3. First of all, let $u \in W^{1, p}(] 0,1\left[; \mathbb{R}^{m}\right)$ be fixed, we can easily see that:

$$
\tilde{I}(u) \leq \int_{0}^{1} f^{* *}\left(u^{\prime}(x)\right) d x
$$

Thus, by combining with formula (4.6), we infer that:

$$
\tilde{I}(u)=\int_{0}^{1} f^{* *}\left(u^{\prime}(x)\right) d x
$$

for all $u \in W^{1, p}(] 0,1\left[; \mathbb{R}^{m}\right)$.

Let us now consider any $u \in W^{1, q}(] 0,1\left[; \mathbb{R}^{m}\right)$. Since in the case when $\int_{0}^{1} f^{* *}\left(u^{\prime}(x)\right)$ $d x=+\infty$ there is nothing to show, we may also assume that $\int_{0}^{1} f^{* *}\left(u^{\prime}(x)\right) d x$ is finite. Following Lemma 4.3, there exists a sequence $\left(u_{s}\right)_{s \in \mathbb{N}} \in C^{\infty}\left([0,1] ; \mathbb{R}^{m}\right)$, such that when $s \rightarrow+\infty$ the following holds:

$$
\left\|u_{s}-u\right\|_{W^{1, q}(] 0,1\left[; \mathbb{R}^{m}\right)} \longrightarrow 0, \quad \int_{0}^{1} f^{* *}\left(u_{s}^{\prime}(x)\right) d x \longrightarrow \int_{0}^{1}\left(u^{\prime}(x)\right) d x .
$$

From formula (4.8), we have:

$$
\tilde{I}\left(u_{s}\right)=\int_{0}^{1} f^{* *}\left(u_{s}^{\prime}(x)\right) d x
$$

for all $s \in \mathbb{N}$.

By passing to the inferior limit, we obtain:

$$
\tilde{I}(u) \leq \liminf _{s \rightarrow+\infty} \tilde{I}\left(u_{s}\right)=\lim _{s \rightarrow+\infty} \int_{0}^{1} f^{* *}\left(u_{s}^{\prime}(x)\right) d x=\int_{0}^{1} f^{* *}\left(u^{\prime}(x)\right) d x .
$$

This implies that:

$$
\tilde{I}(u) \leq \int_{0}^{1} f^{* *}\left(u^{\prime}(x)\right) d x
$$

Finally, note that this generalization will be not true in the general multidimensional case $n, m \geq 2$, see Ball and Murat [5], Fonseca and Malý [18] and Bouchitté et al. [9].

\section{ApplicAtions TO NONLINEAR ELASTICITY}

Some nonlinear models for elastic thin structures were developed using variationals methods in the work of Acerbi et al. [1] for strings, Anzellotti et al. [2], Le Dret and Raoult [21, 22] for membranes with finite energy, Ben Belgacem $[7,8]$ for a generalization to more realistic cases.

Let $W$ be the stored energy function of an homogeneous hyperelastic material. As is usual in nonlinear elasticity, we assume that:

i) $W: M^{3 \times 3} \rightarrow \overline{\mathbb{R}}$ is continuous and bounded below,

ii) $W(F)=+\infty$ if and only if $\operatorname{det} F \leq 0$ and $W(F) \rightarrow+\infty$ when $\operatorname{det} F \rightarrow 0^{+}$.

The latter restriction is imposed to prevent local interpenetration of matter. We now give two applications. 
5.1. Elastic membranes $(n=2, m=3)$

As in $[21,22]$ and $[7,8]$, we define for $F \in M^{3 \times 2}$ the auxiliary function:

$$
W_{0}(F)=\inf _{z \in \mathbb{R}^{3}} W((F \mid z)),
$$

where $(F \mid z)$ denotes the matrix of $M^{3 \times 3}$ whose third column is $z$.

From hypothesis ii) it follows that

$$
W_{0}(F)=+\infty \text { if and only if } \operatorname{rank} F<2 .
$$

Which implies that that the effective domain of $W_{0}$ is $\mathcal{D}_{e}\left(W_{0}\right)=\left\{F \in M^{3 \times 2}\right.$; rank $\left.F=2\right\}$. The density of the class of matrices of maximal rank in $M^{3 \times 2}$ implies that $\mathcal{D}_{e}\left(W_{0}\right)$ is everywhere dense and condition $\left(\mathrm{H}_{1}\right)$ is satisfied with $O_{W_{0}}=\mathcal{D}_{e}\left(W_{0}\right)$.

Now, since $W$ is continuous, it follows that $W_{0}$ is upper semi-continuous on $M^{3 \times 2}$. This implies that $R_{i} W_{0}$ is upper semi-continuous for all $i \in \mathbb{N}$ and condition $\left(\mathrm{H}_{2}\right)$ is satisfied.

To obtain the growth assumption on $R W_{0}$, let us suppose that $W$ satisfies the additional hypothesis for some fixed $p>1$ :

$$
\forall \delta>0, \exists C_{\delta}>0 ; \forall F \in M^{3 \times 3} \text { with } \operatorname{det} F \geq \delta, W(F) \leq C_{\delta}\left(1+\|F\|^{p}\right) .
$$

This implies that $W_{0}$ satisfies:

$$
\forall \delta>0, \exists C_{\delta}>0 ; \forall F \in M^{3 \times 2} \text { with } \operatorname{det}\left(F^{T} F\right) \geq \delta, W_{0}(F) \leq C_{\delta}\left(1+\|F\|^{p}\right) .
$$

Now, after some elementary computation, we obtain that:

$$
R_{2} W_{0}(F) \leq C\left(1+\|F\|^{p}\right) \forall F \in M^{3 \times 2},
$$

for some fixed $C>0$.

This yields, that $R W_{0}$ satisfies:

$$
R W_{0}(F) \leq C\left(1+\|F\|^{p}\right) \text {, for all } F \in M^{3 \times 2} .
$$

Let us now consider the functional:

$$
I(u)=\int_{\omega} W_{0}(\nabla u(x)) d x,
$$

Using our relaxation result, we obtain that:

$$
\bar{I}(u)=\int_{\omega} Q\left[R W_{0}\right](\nabla u(x)) d x,
$$

which is the nonlinear membrane internal energy.

5.2. Elastic strings $(n=1$ and $m=3$ )

Using the same approach as the case of membranes, we let

$$
W_{0}(z)=\inf _{a, b \in \mathbb{R}^{3}} W((z|a| b)),
$$

and the nonlinear string energy is given by

$$
\bar{I}(u)=\int_{0}^{1} W_{0}^{* *}\left(u^{\prime}(x)\right) d x .
$$


Now, we show how our relaxation result allow us to consider a large class of hyperelastic materials, such as those of Ogden.

For a given matrix $F \in M^{3 \times 3}$, let us denote by $0 \leq v_{1} \leq v_{2} \leq v_{3}$ its singular values. The stored energy function of an Ogden material is as follows:

$$
W(F):=\sum_{i=1}^{M} a_{i}\left(v_{1}^{\alpha_{i}}+v_{2}^{\alpha_{i}}+v_{3}^{\alpha_{i}}\right)+\sum_{j=1}^{N} b_{j}\left(v_{1}^{\beta_{j}} v_{2}^{\beta_{j}}+v_{1}^{\beta_{j}} v_{3}^{\beta_{j}}+v_{2}^{\beta_{j}} v_{3}^{\beta_{j}}\right)+\Gamma\left(v_{1} v_{2} v_{3}\right),
$$

where $a_{i}>0, b_{j}>0, \alpha_{i} \geq 1, \beta_{j} \geq 1$ and $\Gamma$ is convex.

Let us now restrict ourself to the simple case; $M=1, N=1$ and $\Gamma(t)=t^{-\gamma}$ for some constant $\gamma>0$. An easy computation will lead to the following bound below and growth assumptions:

$$
\exists c, c_{0}>0 ; c\|F\|^{\alpha}-c_{0} \leq W(F), \forall F \in M_{+}^{3 \times 3},
$$

and

for each $\delta>0, \exists C(\delta)>0 ; W(F) \leq C(\delta)\left(1+\|F\|^{\alpha}+\|F\|^{2 \beta}\right)$ whenever $\operatorname{det} F \geq \delta$.

The auxiliary function $W_{0}$ should satisfy a similar assumptions with the same exponents. Thus, the exponents of growth and coerciveness for $W_{0}$ that we obtain are different in general. However, our Theorem 4.1 works for such functions.

I would like to thank Professor H. Le Dret for useful discussions and his encouragements during the completion of this work.

\section{REFERENCES}

[1] E. Acerbi, G. Buttazzo and D. Percivale, A variational definition of the strain energy for an elastic string. J. Elasticity 25 (1991) 137-148.

[2] E. Acerbi and N. Fusco, Semicontinuity problems in the calculus of variations. Arch. Rational Mech. Anal. 86 (1984) 125-145.

[3] G. Anzellotti, S. Baldo and D. Percivale, Dimension reduction in variational problems, asymptotic development in $\Gamma$-convergence, and thin structures in elasticity. Asymptot. Anal. 9 (1994) 61-100.

[4] J.M. Ball, Convexity conditions and existence theorems in nonlinear elasticity. Arch. Rational Mech. Anal. 63 (1977) $337-403$.

[5] J.M. Ball and F. Murat, $W^{1, p}$-quasiconvexity and variational problems for multiple integrals. J. Funct. Anal. 58 (1984) $225-253$.

[6] J.M. Ball and R.D. James, Fine phase mixtures as minimizers of energy. Arch. Rational Mech. Anal. 100 (1987) 13-52.

[7] H. Ben Belgacem, Une méthode de $\Gamma$-convergence pour un modèle de membrane non linéaire. C. R. Acad. Sci. Paris. Sér. I Math. (1996) 845-849.

[8] H. Ben Belgacem, Modélisation de structures minces en élasticité non linéaire. Thèse de l'Université Pierre et Marie Curie, Paris (1996).

[9] G. Bouchitté, I. Fonseca and J. Malý, The effective bulk energy of the relaxed energy of multiple integrals below the growth exponent. Proc. Roy. Soc. Edinburgh Sect. A 128 (1998) 463-479.

[10] P.G. Ciarlet, Mathematical Elasticity. Vol. I: Three-dimensional Elasticity. North-Holland, Amesterdam (1988).

[11] B. Dacorogna, Quasiconvexity and relaxation of non convex problems in the calculus of variations. J. Funct. Anal. 46 (1982) $102-118$.

[12] B. Dacorogna, Remarques sur les notions de polyconvexité, quasiconvexité et convexité de rang 1. J. Math. Pures Appl. 64 (1985) 403-438.

[13] B. Dacorogna, Direct Methods in the Calculus of Variations. Springer-Verlag, Berlin, Appl. Math. Sci. 78 (1989).

[14] B. Dacorogna and P. Marcellini, General existence theorems for Hamilton-Jacobi equations in the scalar and vectoriel cases. Acta Math. 178 (1997) 1-37.

[15] I. Ekeland and R. Temam, Analyse convexe et problèmes variationnels. Dunod, Paris (1974).

[16] I. Fonseca, The lower quasiconvex envelope of the stored energy for an elastic crystal. J. Math. Pures Appl. 67 (1988) $175-195$.

[17] I. Fonseca, Variational techniques for problems in materials science. Progr. Nonlinear Differential Equations Appl. 25 (1996) $162-175$.

[18] I. Fonseca and J. Malý, Relaxation of multiple integrals below the growth exponent. Ann. Inst. H. Poincaré 14 (1997) $309-338$.

[19] R.V. Kohn and G. Strang, Explicit relaxation of a variational problem in optimal design. Bull. Amer. Math. Soc. 9 (1983) 211-214. 
[20] R.V. Kohn and G. Strang, Optimal design and relaxation of variational problems I, II and III. Comm. Pure Appl. Math. 39 (1986) 113-137, 139-182, 353-377.

[21] H. Le Dret and A. Raoult, Le modèle de membrane non linéaire comme limite variationnelle de l'élasticité non linéaire tridimensionnelle. C. R. Acad. Sci. Paris Sér. I Math. (1993) 221-226.

[22] H. Le Dret and A. Raoult, The nonlinear membrane model as variational limit of three-dimensional nonlinear elasticity. $J$. Math. Pures Appl. 74 (1995) 549-578.

[23] P. Marcellini, Approximation of quasiconvex functions and lower semicontinuity of multiple integrals. Manuscripta Math. 51 (1985) $1-28$

[24] P. Marcellini, On the definition and weak lower semicontinuity of certain quasiconvex integrals. Ann. Inst. H. Poincaré 3 (1986) 391-409.

[25] C.B. Morrey Jr., Quasi-convexity and the lower semi-continuity of multiple integrals. Pacific J. Math. 2 (1952) $25-53$.

[26] C.B. Morrey Jr., Multiple Integrals in the Calculus of Variations. Springer, Berlin (1966).

[27] S. Müller, Variational models for microstructure and phase transitions, to appear in Proc. C.I.M.E. summer school "Calculus of variations and geometric evolution problems". Cetraro (1996).

[28] R.W. Ogden, Large deformation isotropic elasticity: On the correlation of the theory and experiment for compressible rubberlike solids. Proc. Roy. Soc. London Ser. A 328 (1972).

[29] E.T. Rockafellar, Convex Analysis. Princeton University Press (1970).

[30] L. Tartar, Compensated Compactness and Applications to Partial Differential Equations, in Nonlinear Analysis and Mechanics, Heriot-Watt Symp. Vol. IV, R.J. Knops Ed. Pitman, London (1979).

[31] V. Zhikov, Lavrentiev phenomenon and homogenization for some variational problems. C. R. Acad. Sci. Paris Sér. I Math. (1993) 435-439. 